\title{
Concurrent validity of an estimator of weekly alcohol consumption (EWAC) based on the extended AUDIT
}

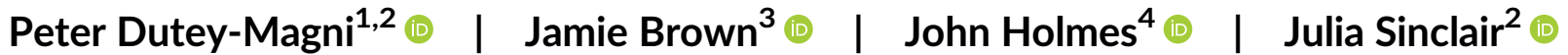

${ }^{1}$ Institute of Health Informatics, University College London, London, UK

${ }^{2}$ Faculty of Medicine, University of Southampton, Southampton, UK

${ }^{3}$ Institute of Epidemiology and Health Care, University College London, London, UK

${ }^{4}$ School of Health and Related Research, University of Sheffield, Sheffield, UK

\section{Correspondence}

Peter Dutey-Magni, Institute of Health Informatics, University College London, 222 Euston Road, London, NW1 2DA, UK.

Email: p.dutey-magni@ucl.ac.uk

\section{Funding information}

Medical Research Council, Grant/Award Number: MR/P016960/1; National Institute for Health Research, Grant/Award Number: SPHR-SWP-ALC-WP5; Public Health Research Programme, Grant/Award Number: 15/63/01

\begin{abstract}
Background and Aims: The three-question Alcohol Use Disorders Identification Test (AUDIT-C) is frequently used in healthcare for screening and brief advice about levels of alcohol consumption. AUDIT-C scores (0-12) provide feedback as categories of risk rather than estimates of actual alcohol intake, an important metric for behaviour change. The study aimed to (i) develop a continuous metric from the Extended AUDIT-C expressed in United Kingdom (UK) units (8 g pure ethanol), offering equivalent accuracy, and providing a direct estimator of weekly alcohol consumption (EWAC) and (ii) evaluate the EWAC's bias and error using the graduated-frequency (GF) questionnaire as a reference standard of alcohol consumption.
\end{abstract}

Design: Cross-sectional diagnostic study based on a nationally-representative survey.

Settings: Community dwelling households in England.

Participants: A total of 22404 household residents aged $\geq 16$ years reporting drinking alcohol at least occasionally.

Measurements: Computer-assisted personal interviews consisting of (i) AUDIT questionnaire with extended response items (the 'Extended AUDIT') and (ii) GF. Primary outcomes were: mean deviation <1 UK unit (metric of bias); root-mean-square deviation <2 UK units (metric of total error) between EWAC and GF. The secondary outcome was the receiver operating characteristic area under the curve for predicting alcohol consumption in excess of 14 and 35 UK units.

Findings: EWAC had a positive bias of 0.2 UK units $(95 \% \mathrm{Cl}=0.08,0.4)$ compared with GF. Deviations were skewed: whereas the mean error was \pm 11 UK units/week [9.5, 11.9], in half of participants the deviation between EWAC and GF was between 0 and $\pm 2.1 \mathrm{UK}$ units/week. EWAC predicted consumption in excess of $14 \mathrm{UK}$ units/week with a significantly greater area under the curve $(0.918[0.914,0.923])$ than AUDIT-C $(0.870$ $[0.864,0.876])$ or the full AUDIT $(0.854[0.847,0.860])$.

Conclusions: A new estimator of weekly alcohol consumption, which uses answers to the Extended AUDIT-C, meets the targeted bias tolerance. It is superior in accuracy to AUDIT-C and the full 10-item AUDIT when predicting consumption thresholds, making it a reliable complement to the Extended AUDIT-C for health promotion interventions. 


\section{KEYWORDS}

Alcohol consumption, alcohol use disorder, diagnostic, preventive health services, screening

programs, self-report

\section{INTRODUCTION}

Alcohol consumption is responsible for $5 \%$ of disability-adjusted life years [1]. This burden extends far beyond the health burden of alcohol use disorders, as defined in the International Statistical Classification of Diseases (ICD-10 F10.1/F10.2 [2]) or the Diagnostic and Statistical Manual of Mental Disorders [3]. Clinical guidelines aiming to prevent [4], treat and reduce [5] harm from alcohol consumption recommends systematic screening for alcohol consumption using validated clinical tools. However, conceptual differences (exemplified by the diagnostic classifications above) remain in how best to diagnose, measure and communicate harm [6].

A global standard has emerged in the 10-item Alcohol Use Disorders Identification Test (AUDIT) [7]. The shorter three-item AUDIT-C focusses on consumption, and has equivalent predictive capability [8]. AUDIT-C is easy to use, making it an attractive choice for alcohol screening and brief interventions in healthcare [9] and other settings [10]. AUDIT-C exhibits two characteristics.

1. Ceiling effect: AUDIT-C's maximum response options for alcohol consumption frequency and quantity are heavily right-censored (Table 1). This creates a ceiling effect making the AUDIT-C poorly responsive to change in individuals with a high baseline score (up to reductions of $30 \%$; e.g. frequency of drinking down from 7 to 5 days or quantity down from 16 to 11 drinks per day).
2. AUDIT score interpretation: the ordinal scores produced by the AUDIT-C (range: 0-12) and the full AUDIT (range: 0-40) are multidimensional measures of alcohol risk. To date, most brief intervention models involve dichotomising AUDIT scores, on the basis of complex diagnostic accuracy studies [11], at cut-offs that vary internationally [12]. In practice, this may contribute to healthcare professionals lacking confidence in discussing alcohol risks and consumption [13-16], and needing to be trained to deliver feedback $[13,14]$. Evidence also suggests that patients' understanding of alcohol risks overlaps loss of control more than alcohol consumption $[17,18]$. In response, some academic models of alcohol care advocate the framing of brief interventions around the continuum of alcohol use [19] rather than thresholds, because these can trigger stigma related to loss of control [20].

The 'Extended AUDIT-C' addresses the first characteristic thanks to a greater range of response options on quantity and frequency (Table 1). It has been used in the United Kingdom (UK) as part of two trials [21,22] and one continuous household survey [23] to measure characteristics of consumption that could not have been measured with the right-censored AUDIT-C.

The present study proposes to address the second of these characteristics. It aims to develop and validate an estimator of weekly alcohol consumption (EWAC) computed from the Extended AUDIT-C. Although retaining the Extended AUDIT-C questionnaire's alcohol use disorders diagnostic capabilities, the EWAC is intended to facilitate the delivery of screening and brief interventions by converting

T A B LE 1 Comparison of AUDIT-C and Extended AUDIT-C.

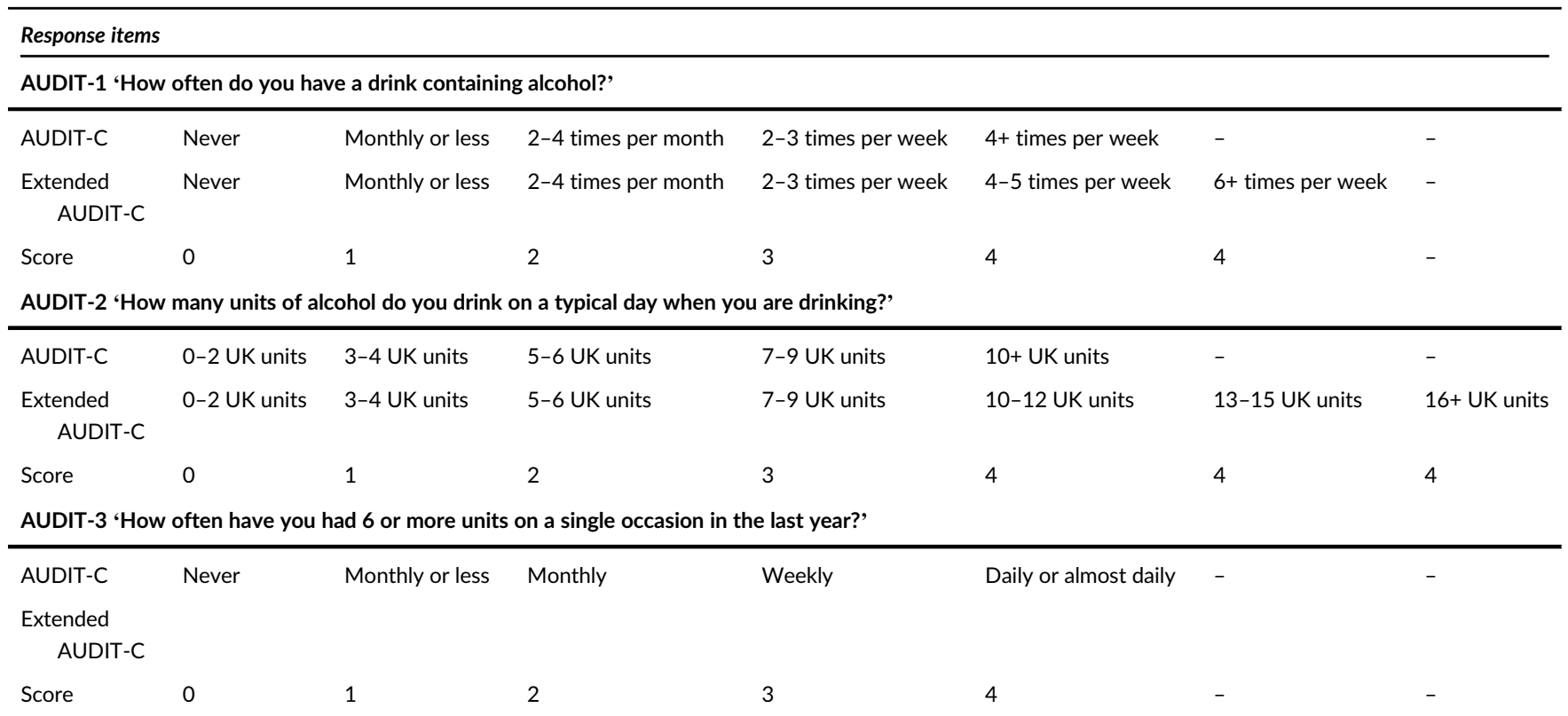


Extended AUDIT-C responses into a continuous and direct measure of alcohol consumption that does not require additional screening questions. Measuring alcohol consumption is a crucial part of behaviour change techniques (self-monitoring, feedback on behaviour, social comparison) commonly used in self- [10] and clinician-administered $[9,24]$ interventions, and is encouraged as a metric of the continuum of alcohol use [19].

\section{METHODS}

\section{Participants}

Data originate from baseline measures in waves 110-133 (November 2015-October 2017) of the Alcohol Toolkit Study, a repeated crosssectional survey of residents of private English households aged $\geq 16$ years. Each month, census output areas averaging 300 households were selected by stratified random sampling. Interviewers travelled to their designated area and approached households using quota sampling [23]. Respondents participated in a computer-assisted personal interview.

\section{Measures}

Index measurements underpinning the EWAC were the three questions making up the Extended AUDIT-C (Data S2), in which participants described their drinking during the last 6 months.

The reference standard used is the Alcohol Toolkit Study graduated-frequency (GF) schedule (Data S3), in which participants described how many times they consumed given quantities of alcohol during the last 4 weeks [25]. The GF schedule's main advantage lies in measuring occasional heavy consumption, which can constitute an important proportion of total consumption.

Other reference estimates were used, this time for aggregate comparisons. The 2014 per-capita alcohol retail sales [26] captured all alcohol produced/processed in or imported to England for sale or consumption. We also used data from 6606 household residents aged $\geq 18$ years participating in the 2011 Health Survey for England [27]. The year 2011 was chosen in deviation from the registered protocol [28]: on that particularly year, the computer-assisted interviewer-led beverage-specific quantity-frequency questionnaire was accompanied by a prospective 7-day diary [29]. The diary reference standard was deemed more informative to an international audience, and offered a direct point of comparison with past research [30-32].

\section{Estimating alcohol consumption}

To estimate alcohol consumption from Extended AUDIT-C responses, we use methods developed for quantity-frequency- variability instruments [33]. For every individual $i$, the EWAC is computed as the product of $F_{i}$ and $Q_{i}$ (AUDIT questions 1 and 2 , respectively) adjusted with the frequency of intense drinking $V_{i}$ (AUDIT-3) :

$$
\mathrm{EWAC}_{i}=F_{i} Q_{i}+V_{i}
$$

where $b$ denotes the mean units of alcohol consumed in an intense drinking day.

Coefficients $F, Q, V$ and $b$ are unknown. In this study, two sets of candidate coefficients are considered:

1. AUDIT response item interval midpoint (e.g. 2.5 for ' 2 to 3 times per week') and

2. coefficients estimated empirically from a sample of individuals with measurements of Extended AUDIT-C and GF, using a hierarchical Bayesian response model with the estimating equation $\mathrm{GF}_{i}=F_{i} Q_{i}+V_{i} b+e_{i}$, where $e$ denotes independently normally distributed errors. We set parabola-shaped informative priors on coefficients $F, Q$, and V. Details on model fitting, convergence evaluation and prior tuning are reported in Data S1.

\section{Analyses}

The protocol was pre-registered [28]. Results are reported in UK alcohol units ( $8 \mathrm{~g}$ or $10 \mathrm{~mL}$ of pure alcohol). Analyses were conducted in $\mathrm{R}$ [34-36] and all computer scripts are available online [37].

Participants were included in the analysis if they completed both the Extended AUDIT and the GF questionnaires. Of 40832 participants, 14408 (35\%) reported 'never' consuming alcohol in AUDIT question 1 and were not asked any further AUDIT or GF questions. A further 175 (0.4\%) did not have valid AUDIT-C answers. Finally, 3876 participants (9\%) who did not have a valid GF alcohol consumption record were excluded. These GF data were assumed to be missing at random conditionally on the Extended AUDIT-C responses after a sensitivity analysis (Data S1).

Valid observations ( $n=22373$ ) were separated into two datasets:

1. the training dataset $(n=6642)$ consisted of a $30 \%$ subset of participants drawn using stratified random sampling, ensuing a balanced representation by sex, age, ethnic group and AUDIT-C risk level. It was used to estimate coefficients underpinning the EWAC (Data S1).

2. The validation dataset consisted of the remaining participants ( $n=15731)$ and was used to evaluate the EWAC's bias and precision. In subgroup validation analyses using additional variables (e.g. education, smoking status), a further 358 of 15731 
observations (2.3\%) assumed to be missing at random were excluded.

\section{Overall bias and error}

The agreement between the EWAC and the GF was quantified in the validation dataset:

1. bias was estimated by the mean deviation (MD) to the reference standard $M D=n^{-1} \sum_{i=1}^{n}(E W A C-G F)$. We tested the hypothesis that the MD does not exceed 1 UK unit using a two-sided $t$ test.

2. Precision was estimated by the root-mean-square deviation $\mathrm{RMSD}=\sqrt{n^{-1} \sum_{i=1}^{n}(E W A C-G F)^{2}}$, a measure of total error capturing both bias and random deviation from the reference standard. For example, an RMSD of 2 signifies that the EWAC is on average within \pm 2 UK units of the reference standard. We tested the hypothesis that the RMSD does not exceed 2 UK units using a one-sided $\chi^{2}$ test.

Two sets of candidate coefficients were considered (see 'Estimating alcohol consumption' section). We only report findings for the candidate set producing the lowest bias and error.

\section{Subgroup bias and error}

Multivariate regression models tested whether the EWAC's bias and precision varied across population subgroups:

1. the simple deviation (EWAC - GF) was regressed in a linear model to test subgroup differences in MD; and

2. the square deviation (EWAC-GF) ${ }^{2}$ was regressed in a log-transformed linear model to test subgroup differences in the geometric mean-square deviation. Model coefficients were then back-transformed (square root of the exponential) into relative RMSD estimates; these are interpreted as the ratio of the subgroup RMSD to the reference RMSD, a ratio $>1$ indicating worse precision than in the reference category.

Both models (Data S5, see Table S5.1) included the following predictors: sex by age group, ethnic group, highest educational qualification, religion, and smoking status. Additional models (Data S5, see Table S5.2) were fitted solely in respondents with an AUDIT-C score $\geq 5$ or an AUDIT score $\geq 8$, for whom additional characteristics were recorded during interview: favourite drink (beer, wine, spirits alone, mixed spirits, cider, other); and whether the respondent had attempted to restrict alcohol intake in the last 12 months.

\section{Receiver operating characteristics}

We tested the EWAC's superiority to the traditional AUDIT and AUDIT-C scores in predicting consumption exceeding 14 or 35 UK units/week. These correspond to UK thresholds for characterising alcohol use as 'increasing risk' (predicted by an AUDIT-C score of $5-7$ ), and 'higher risk' (AUDIT-C score $\geq 8$ ), which is above 35 units for women and 50 units for men [38]. We tested the hypothesis that the EWAC has an identical receiver operating characteristic full area under the curve (AUC) to the AUDIT-C and the full AUDIT scores using nonparametric paired AUC tests [39]. AUDIT-C and AUDIT scores were calculated from the Extended AUDIT by capping the contribution of each question to 4 .

\section{Aggregate concurrent validity}

We compared the empirical cumulative distributions of (i) the EWAC computed in the Alcohol Toolkit Study; (ii) the GF estimator in the Alcohol Toolkit Study; (iii) the beverage-specific estimator in the 2011 Health Survey for England; and (iv) the prospective diary estimator in the 2011 Health Survey for England in adults aged $\geq 18$ years. A $\chi^{2}$ test of homogeneity of distributions (i) and (iii) was performed on contingency tables of 13 drinking consumption intervals in UK units/week (]0,5]; ]5,10]; ... ]30,35]; ]35,45]; ]45,55]; ] 55,65]; ]65,75]; ]75,100]; ]100,200]). We report the proportions of on-trade and off-trade alcohol sales [26] accounted for by each method.

Poststratification survey weights adjusted for nonresponse bias in sources (i-iii), and self-selection into prospective diary data collection in source (iv).

\section{RESULTS}

\section{Bias and precision}

EWAC coefficients estimated empirically (Data S1, Data S4) had smaller bias and error and were used for the remainder of the analysis. With those, the EWAC's Pearson's correlation with GF was estimated at $r=0.72[0.71,0.72]$ (Kendall's rank correlation $\tau=0.63$ ).

The mean deviation (MD) was 0.2 alcohol units/week $(95 \% \mathrm{Cl}=$ $0.08,0.4)$. This bias is smaller than the preregistered \pm 1 -unit bias tolerance $(P=1.000)$.

The RMSD, at 10.7 units/week $(95 \% \mathrm{Cl}=9.5,11.9)$, was significantly greater than the pre-registered 2 -unit total error tolerance $(P<$ 0.001 ), suggesting that the EWAC falls on average 11 units away from the GF reference standard.

However, there was substantial variation in RMSD; in $50 \%$ of participants, the EWAC fell within \pm 2.1 UK units of the GF weekly consumption estimate. RMSD was proportional to alcohol consumption, amounting to $\sim 50 \%$ of the EWAC value (Table 2). 
TA B LE 2 RMSD between EWAC and GF schedule by alcohol consumption bracket $(n=15731)$.

\begin{tabular}{lrcc}
\hline EWAC value (UK units/week) & $n$ & RMSD [95\% CI] & $\begin{array}{c}\text { Participants with GF contained in } \\
\text { [EWAC } \times 0.5 ; \text { EWAC } \times 1.5] \text { interval (\%) }\end{array}$ \\
\hline$[0,5[$ & 6927 & $3.1[2.7-3.5]$ & $3375(48.7)$ \\
{$[5,10[$} & 3589 & $10.0[3.8-13.5]$ & $2127(59.3)$ \\
{$[10,20[$} & 3363 & $12.4[9.3-14.9]$ & $2330(69.3)$ \\
{$[20,30[$} & 1010 & $15.5[12.6-17.9]$ & $736(72.9)$ \\
{$[30,45[$} & 495 & $19.4[17.0-21.4]$ & $342(69.1)$ \\
{$[45,60[$} & 142 & $27.4[22.1-31.8]$ & $101(71.1)$ \\
{$[60,75[$} & 113 & $25.7[18.9-31.0]$ & $95(84.1)$ \\
{$[75,100[$} & 66 & $40.2[25.1-51.0]$ & $48(72.7)$ \\
{$[100,150[$} & 26 & $77.0[59.2-91.4]$ & $13(50.0)$ \\
All values & 15731 & $10.7[9.4-11.9]$ & $9167(58.3)$ \\
\hline
\end{tabular}

EWAC = estimator of weekly alcohol consumption; GF = graduated frequency; RMSD = root-mean-square deviation

(a)

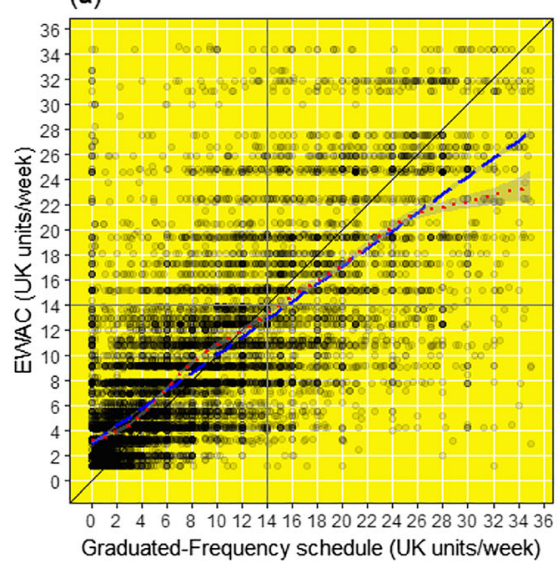

(b)

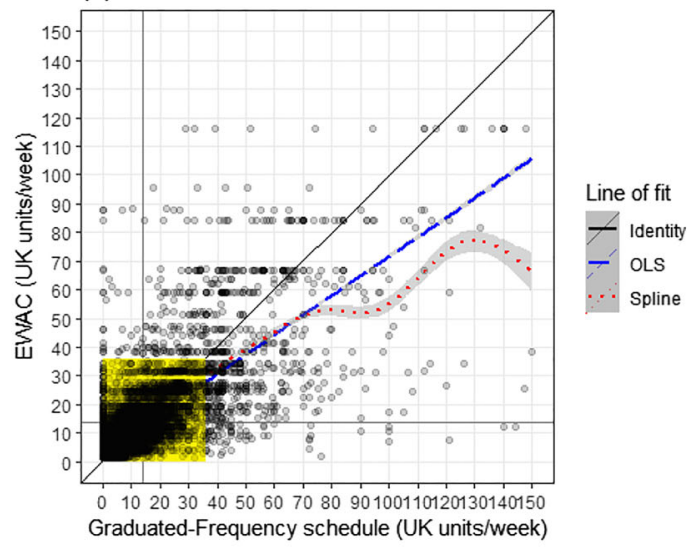

F I G URE 1 Plots of EWAC against GF in (a) low/increasing risk respondents ( $n=15008)$ and (b) all respondents $(n=15731)$

Therefore, an interval defined as the EWAC $\pm 50 \%$ (e.g. '2-6 units/ week' for an EWAC of 4; '10-30 units/week' for an EWAC of 20) contained the reference standard for over half $(58 \%)$ of the individuals.

Plots of EWAC against GF (Figure 1) indicate a slight positive bias for consumptions up to 10-14 units/week, and a slight negative bias beyond. The EWAC only starts losing granularity above 70 units/week (99th percentile of its distribution), where it provides just 6 possible values (82; 83; 92; 93; 100; 125 units/week; see Figure 1b).

Subgroup analyses are reported in Data S5. A very modest proportion of variation in bias and precision $(<5 \%)$ can be attributed to sociodemographic variables under examination. This indicates a relative homogeneity in precision in bias, to one exception. The EWAC appears to overestimate consumption by 2 to 3 UK units in groups with the lowest average consumption: non-British White, Black and Other ethnic groups.

\section{Receiver operating characteristics}

We examined the EWAC's ability to predict consumption exceeding 14 or 35 UK units/week. The full areas under the receiver operating characteristic curves (AUC, Figure S1) are presented along sensitivity and specificity at the best thresholds in Tables 3 and 4 .

At 14 UK units/week (increasing-risk) EWAC increases the AUC by 5 percentage points compared to the AUDIT-C score $(P<0.001)$; and 7 percentage points compared to the full AUDIT score $(P<$ 0.001). The cut-off maximising the sum of specificity and sensitivity on the EWAC is 10 units/week. The sensitivity at this threshold is identical to AUDIT-C, but specificity gains 13 percentage points. Using the nominal cut-off of 14 units/week on the EWAC raises specificity to 0.928 , at the cost of a reduction in sensitivity to 0.687 (Table 3).

At 35 units/week (higher-risk) EWAC provides small increases in AUC compared with the AUDIT-C score $(P<0.001)$ and the full 
T A B L E 3 Receiver operating characteristics of AUDIT-C score and EWAC for consumption $\geq 14$ UK units or $112 \mathrm{~g} /$ week $(n=15731$ ).

\begin{tabular}{|c|c|c|c|c|c|}
\hline Index test & Full area under the curve & $95 \% \mathrm{Cl}$ & Best threshold & Sensitivity & Specificity \\
\hline Full AUDIT score & 0.854 & {$[0.847,0.860]$} & 5.5 & 0.792 & 0.751 \\
\hline
\end{tabular}

Note: The best threshold refers the cut-off value that maximises the sum of sensitivity and specificity.

EWAC = estimator of weekly alcohol consumption.

T A B L E 4 Receiver operating characteristics of AUDIT-C score and EWAC for consumption $\geq 35$ UK units or $280 \mathrm{~g} /$ week $(n=15731)$.

\begin{tabular}{|c|c|c|c|c|c|}
\hline Index test & Full area under the curve & $95 \% \mathrm{Cl}$ & Best threshold & Sensitivity & Specificity \\
\hline Full AUDIT score & 0.900 & {$[0.890,0.910]$} & 6.5 & 0.905 & 0.743 \\
\hline EWAC & 0.934 & {$[0.925,0.943]$} & 16.8 & 0.862 & 0.865 \\
\hline
\end{tabular}

Note: The best threshold refers the cut-off value that maximises the sum of sensitivity and specificity.

EWAC = estimator of weekly alcohol consumption.

T A B LE 5 Summary statistics on alcohol consumption in England in residents aged 18 years and over (excluding abstainers).

\begin{tabular}{|c|c|c|c|c|c|}
\hline Study & Mean (UK units/week) & Median (UK units/week) & Variance & $n$ & $\%$ of alcohol sold \\
\hline HSE prospective diary & 13.0 & 8.0 & 264.7 & 4640 & 67.6 \\
\hline ATS GF & 8.5 & 5.2 & 234.6 & 15556 & 43.9 \\
\hline ATS EWAC & 9.3 & 5.2 & 145.9 & 18140 & 48.2 \\
\hline Retail sales & 19.3 & - & - & - & 100.0 \\
\hline
\end{tabular}

EWAC = estimator of weekly alcohol consumption; GF = graduated-frequency; HSE = health survey for England.

AUDIT score $(P<0.001)$. The best cut-off for detecting consumption in excess of 35 units/week using the EWAC was 17 units/week (Table 4).

\section{Empirical distribution}

Table 5 estimates adult residents' total alcohol consumption in England using four different sources, and compares them with alcohol retail sales. The Health Survey for England exhibits the highest estimates and coverage of alcohol sales. EWAC amounts to $71 \%$ of the total consumption estimated by the Health Survey for England's prospective diary and $48 \%$ of retail sales.

Figure 2 suggests that the EWAC, like the Alcohol Toolkit Study (ATS) GF, estimates a greater prevalence of lower-risk ( $\leq 14$ units/ week) and increasing-risk alcohol use than the Health Survey for England. It shows a clear departure between the EWAC and the Health Survey for England's beverage-specific questionnaire, as evidenced by the homogeneity test $\left(\chi_{12}^{2}=914.8, P<0.001\right)$.

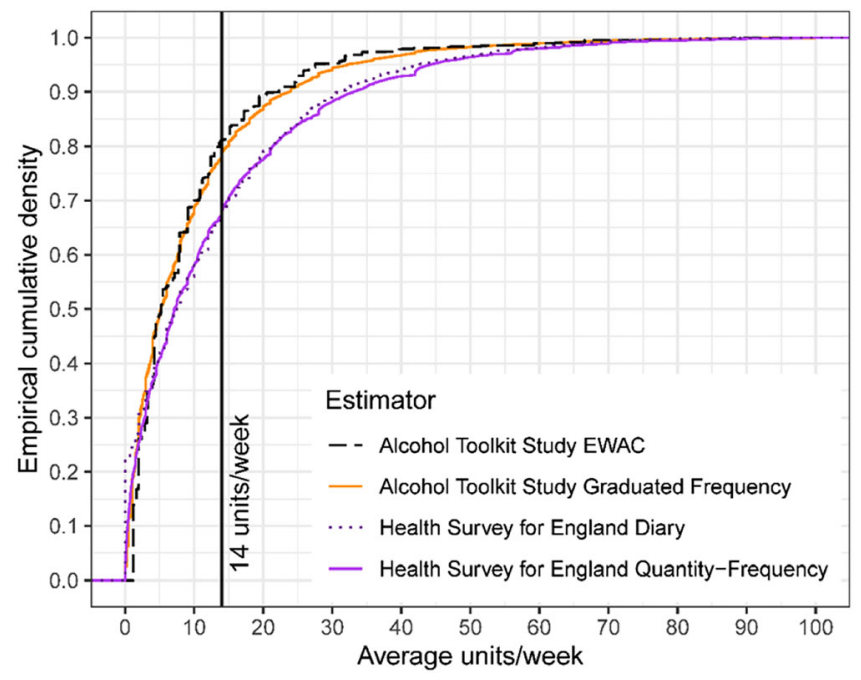

FIG URE 2 Empirical cumulative distribution function of weekly alcohol consumption in England according to four alcohol schedules in residents aged 18 years and over 


\section{DISCUSSION}

\section{Main findings}

We developed a continuous EWAC using a 6-month Extended AUDIT-C. When compared with a 4-week GF reference standard, we found EWAC overestimates alcohol consumption by 0.2 UK units (95\% Cl:0.08, 0.4), well under the pre-registered \pm 1 UK unit bias tolerance. We also attempted to measure how precise the EWAC is; in $50 \%$ of participants, the EWAC falls up to 2 UK units away from the GF measure, and an interval built as EWAC $\pm 50 \%$ contains the GF measures in $58 \%$ of participants.

EWAC is superior to both the AUDIT-C and the full AUDIT scores in predicting GF exceeding 14 units/week (AUC = 0.92) and 35 units/ week ( $A \cup C=0.93$ ). This places the EWAC among the bestperforming diagnostic tools examined in the most recent systematic review [11]. At the 14-unit threshold, an EWAC $\geq 10$ cut-off has a sensitivity of 0.87 , compared to a 0.75 for an AUDIT-C $\geq 6$ cut-off, without losing specificity.

\section{Potential applications}

Being equivalent to the AUDIT-C in speed and international standardisation, the EWAC may be suitable for use in any clinical setting to support brief interventions and to feed back a reliable interval estimate of alcohol consumption (e.g. '6-18 units/week' or '50-140 g/ week'). The EWAC is available as a web app at https://ewac.netlify. app along with resources to facilitate implementation ( $R$ software package and spreadsheets).

Assessment of alcohol consumption is not well embedded in clinical practice [40]. The EWAC calculator fills a gap in resources by transforming the answers from the Extended AUDIT-C into a direct estimate of an individual's weekly alcohol consumption. This is a more directly accessible metric that should facilitate behaviour change by empowering people to monitor and control their alcohol consumption with-or without-the involvement of healthcare professionals and should be assessed in future evaluations.

Nutt and Rehm $[19,41]$ have argued that alcohol-related harm is best prevented if individuals know their consumption level, and health professionals in all settings can engage patients effectively to manage risks with evidence-based interventions, in a similar way to other risk factors for disease, for example, blood pressure or cholesterol. Yet, knowledge of beverages' alcohol content is generally poor [42], and a survey evaluating the 2016 change in UK alcohol guidelines found that just $8 \%$ of the UK drinkers knew the new recommended limits [43]. The EWAC can support interventions focused on recognising the alcohol content/volume of drinks and recommended low-risk limits.

In addition, the EWAC's dimensional, rather than categorical format, can be useful to position recipients of brief interventions on the continuum of alcohol use [20,44], which may reduce the stigma of loss of control associated with screening-based interventions [17,45]. It can act as a complement, rather than a substitute to the multidimensional quality of AUDIT-C or the full AUDIT.

The EWAC is particularly suitable for digital interventions and healthcare records given that it enables its complex algorithm to be embedded in a way not possible with paper records. The EWAC is already compatible with medical records information models developed in the Systematised Nomenclature of Medicine Clinical Terms (SNOMED CT, alcohol intake [observable entity] [46]) and by the English Royal College of Physicians [47]. Such information can have secondary uses as a variable in other disease risk scores, or to prospectively record long-term alcohol exposure, an important risk factor for a range of medical conditions.

\section{Strengths and limitations}

This paper is the first to (i) develop an EWAC using a well-accepted and validated multidimensional alcohol screening tool such as the AUDIT; and (ii) quantify its bias and precision with respect to a continuous measure of alcohol consumption. One study [48] previously reported mean consumption by AUDIT-C score, but without quantifying bias or precision of such a measure. Others have evaluated the AUDIT-C's accuracy in estimating alcohol consumption, but exclusively in relation to predicting consumption in excess of predefined thresholds [11].

Our study provides strong confidence in the internal and external validity of findings in England on account of the large sample size and extensive range of subgroup analyses reported. Bias was mostly consistent across subgroups examined (age, sex, education, smoking status, and religion), with one exception. The EWAC overestimated alcohol consumption by 2-3 UK units/week in Black/Other ethnic groups. Variation in the sensitivity of AUDIT-C across ethnic groups has previously been noted in the United States [49].

Repurposing a well-known tool such as the AUDIT-C has several advantages. It is already translated in many languages and adapted to the varying standard drink sizes adopted internationally [7]. The Extended AUDIT scores can be converted into traditional AUDIT scores by capping items at 4 , thereby offering a point of comparison with existing evidence. The AUDIT's properties are also well understood in diverse contexts and modes of administration, based on the last 30 years of international research. For instance, a previous study that found the AUDIT-C to be responsive to changes of $70 \mathrm{~g} /$ week [50] can suggest that the EWAC's own responsiveness to change should be equivalent, if not greater than the AUDIT-C's, given the Extended AUDIT-C's additional response items.

We note two main study limitations. First, a longstanding obstacle in alcohol research and treatment lies in the absence of undisputed 'gold standard' or biomarker for objectively determining alcohol consumption. Instead, a number of instruments measure self-reported consumption with varying validity and reliability over different durations. Comprehensive reviews [30-32,51-53] indicate that yesterday recall 
and prospective diaries tend to record higher (and more accurate) alcohol consumption by minimising recall bias, followed by GF measures.

Therefore, the GF reference standard, as all self-reported measures, is imperfect. Although this has no effect on our measure of bias (MD), this may introduce bias into our measure of precision (RMSD): by definition, the reference standard's own independent error will inflate the RMSD. In other words, it is likely that a proportion of the RMSD is attributable to error in the GF measures rather than the EWAC.

Despite this, previous research suggests the EWAC's agreement with GF (Pearson's correlation coefficient $r=0.71$ and Kendall's rank correlation $\tau=0.63$ in the present study) is comparable to the agreement between GF and prospective diaries measured from past studies ( $r \sim 0.86-0.89$ [54,55]; $\tau=0.41$ [51]).

Second, the EWAC's design does not escape all limitations of methods of screening or categorising alcohol use disorders. The conceptualisation of alcohol use disorders is related to, but does not exclusively depend on the amount of alcohol consumed. Since Jellinek's description of 'the disease concept of alcoholism' [56] there have been numerous attempts to categorise the range of phenotypes characterising alcohol use disorders in the absence of any biomarker to 'verify' the presence of a particular pathology. The EWAC, by limiting itself to an estimation of alcohol consumption, is transparent across a wide range of alcohol use disorders, but does not measure the other factors underpinning this complex and heterogeneous condition [6,57].

In conclusion, the EWAC has the potential to support interventions focusing on recognising the alcohol content and volume of drinks. The EWAC's dimensional rather than categorical format may facilitate this while avoiding the stigma sometimes associated with clinical categorisations of alcohol use disorders.

\section{PROTOCOL REGISTRATION}

Dutey-Magni, PF, Sinclair, JMA, Brown, J. 2018. 'Concurrent Validity of an Estimator of Weekly Alcohol Consumption (EWAC) Based on the Extended AUDIT.' OSF. November 12. doi:10.17605/OSF. IO/7WE4M.

\section{ACKNOWLEDGEMENTS}

This research was supported by the Medical Research Council (grant reference MR/P016960/1). The Alcohol Toolkit Study data collection was funded primarily by the National Institute for Health Research (NIHR) School for Public Health Research (grant reference SPHRSWP-ALC-WP5) and Public Health Research Programme (grant reference 15/63/01). The EWAC online calculator development was funded by the Wessex Academic Health Science Network. The views expressed are those of the author(s) and not necessarily those of the NHS, the NIHR or the Department of Health.

\section{DECLARATION OF INTERESTS}

J.B. has received unrestricted research funding to study smoking cessation from companies who manufacture smoking cessation medications. P.D., J.H. and J.S. declare no competing interests.

\section{ETHICS}

This study was approved by the University of Southampton's Faculty of Medicine Ethics Committee (ERGO 44682).

\section{AUTHOR CONTRIBUTIONS}

Peter Dutey-Magni: Conceptualization; formal analysis; funding acquisition; investigation; methodology; software. Jamie Brown: Investigation; methodology; resources. John Holmes: Investigation; methodology; resources. Julia Sinclair: Conceptualization; funding acquisition; investigation; methodology; supervision; validation.

\section{ORCID}

Peter Dutey-Magni (D) https://orcid.org/0000-0002-8942-9836

Jamie Brown (D) https://orcid.org/0000-0002-2797-5428

John Holmes (D) https://orcid.org/0000-0001-9283-2151

Julia Sinclair (D) https://orcid.org/0000-0002-1905-2025

\section{REFERENCES}

1. Shield K, Manthey J, Rylett M, Probst C, Wettlaufer A, Parry CDH, et al. National, regional, and global burdens of disease from 2000 to 2016 attributable to alcohol use: a comparative risk assessment study. Lancet Public Health. 2020;5(1):e51-61. https://doi.org/10. 1016/S2468-2667(19)30231-2

2. World Health Organisation. ICD-10: International statistical classification of diseases and related health problems: Tenth revision. 2004.

3. American Psychiatric Association. Diagnostic and Statistical Manual of Mental Disorders. Washington, DC: American Psychiatric Association; 2013. https://doi.org/10.1176/appi.books.9780890425596

4. National Institute of Health and Care Excellence. Alcohol-use disorders: prevention. Public health guideline [PH24] 2010. https://www. nice.org.uk/guidance/ph24

5. National Institute of Health and Care Excellence. Alcohol-use disorders: diagnosis, assessment and management of harmful drinking and alcohol dependence. Clinical guideline [CG115]. 2011. https://www. nice.org.uk/guidance/cg115

6. Saunders JB, Degenhardt L, Reed GM, Poznyak V. Alcohol use disorders in ICD-11: past, present, and future. Alcohol Clin Exp Res. 2019; 43:1617-31. https://doi.org/10.1111/acer.14128

7. Babor TF, Higgins-Biddle JC, Saunders JB, Monteiro MG. The Alcohol Use Disorders Identification Test. Guidelines for Use in Primary Care. Second ed. Geneva: World Health Organisation, Department of Mental Health; Substance Dependence; 2001. https://www.who. int/publications/i/item/audit-the-alcohol-use-disordersidentification-test-guidelines-for-use-in-primary-health-care

8. Bush K. The AUDIT alcohol consumption questions (AUDIT-C): an effective brief screening test for problem drinking. Arch Intern Med. 1998;158:1789-95. https://doi.org/10.1001/archinte.158.16.1789

9. Kaner EF, Beyer FR, Muirhead C, et al. Effectiveness of brief alcohol interventions in primary care populations. Cochrane Database Syst Rev. 2018;2018. https://doi.org/10.1002/14651858.CD004148. pub4

10. Kaner EF, Beyer FR, Garnett C, Crane D, Brown J, Muirhead C, et al. Personalised digital interventions for reducing hazardous and harmful alcohol consumption in community-dwelling populations. Cochrane Database Syst Rev. 2017. https://doi.org/10.1002/14651858. CD011479.pub2

11. de Meneses-Gaya C, Zuardi AW, Loureiro SR, Crippa JAS. Alcohol use disorders identification test (AUDIT): an updated systematic review of psychometric properties. Psychol Neurosci. 2009;2(1): 83-97. https://doi.org/10.3922/j.psns.2009.1.12 
12. Nadkarni A, Garber A, Costa S, Wood S, Kumar S, MacKinnon N, et al. Auditing the AUDIT: a systematic review of cut-off scores for the alcohol use disorders identification test (AUDIT) in low- and middle-income countries. Drug Alcohol Depend. 2019;202:123-33. https://doi.org/10.1016/j.drugalcdep.2019.04.031

13. Beich A, Gannik D, Malterud K. Screening and brief intervention for excessive alcohol use: Qualitative interview study of the experiences of general practitioners. BMJ. 2002;325:870. https://doi.org/10. 1136/bmj.325.7369.870

14. Johnson M, Jackson R, Guillaume L, Meier P, Goyder E. Barriers and facilitators to implementing screening and brief intervention for alcohol misuse: a systematic review of qualitative evidence. J Public Health. 2011;33:412-21. https://doi.org/10.1093/pubmed/fdq095

15. Hutchings D, Cassidy P, Dallolio E, Pearson P, Heather N, Kaner E. Implementing screening and brief alcohol interventions in primary care: views from both sides of the consultation. Prim Health Care Res Dev. 2006;7:221-9. https://doi.org/10.1191/ $1463423606 p c 2920 a$

16. McCormick KA, Cochran NE, Back AL, Merrill JO, Williams EC Bradley KA. How primary care providers talk to patients about alcohol. J Gen Intern Med. 2006;21(9):966-72. https://doi.org/10.1111/ j.1525-1497.2006.00490.x

17. Khadjesari Z, Stevenson F, Toner P, Linke S, Milward J, Murray E. 'I'm not a real boozer': a qualitative study of primary care patients' views on drinking and its consequences. J Public Health. 2018;41(2):e185-91. https://doi.org/10.1093/pubmed/fdy067

18. O'Donnell A, Hanratty B, Schulte B, Kaner E. Patients' experiences of alcohol screening and advice in primary care: a qualitative study. BMC Fam Pract. 2020;21(1):68. https://doi.org/10.1186/s12875020-01142-9

19. Nutt DJ, Rehm J. Doing it by numbers: a simple approach to reducing the harms of alcohol. J Psychopharmacol. 2014;28(1):3-7. https://doi.org/10.1177/0269881113512038

20. Rehm J, Marmet S, Anderson P, Gual A, Kraus L, Nutt DJ, et al. Defining substance use disorders: do we really need more than heavy use? Alcohol Alcohol. 2013;48(6):633-40. https://doi.org/10. 1093/alcalc/agt127

21. Kaner E, Bland M, Cassidy P, et al. Effectiveness of screening and brief alcohol intervention in primary care (SIPS trial): pragmatic cluster randomised controlled trial. BMJ. 2013;346:e8501-1. https:// doi.org/10.1136/bmj.e8501

22. Crane D, Garnett C, Michie S, West R, Brown J. A smartphone app to reduce excessive alcohol consumption: identifying the effectiveness of intervention components in a factorial randomised control trial. Sci Rep. 2018;8(1):4384. https://doi.org/10.1038/s41598-01822420-8

23. Beard E, Brown J, West R, Acton C, Brennan A, Drummond C, et al. Protocol for a national monthly survey of alcohol use in England with 6-month follow-up: 'The alcohol toolkit study' health behavior, health promotion and society. BMC Public Health. 2015;15(1):230. https://doi.org/10.1186/s12889-015-1542-7

24. Michie S, Whittington C, Hamoudi Z, Zarnani F, Tober G, West R. Identification of behaviour change techniques to reduce excessive alcohol consumption. Addiction. 2012;107(8): 1431-40. https://doi.org/10.1111/j.1360-0443.2012.03845.x

25. Greenfield TK. Ways of measuring drinking patterns and the difference they make: experience with graduated frequencies. J Subst Abuse. 2000;12:33-49. https://doi.org/10.1016/S0899-3289(00) 00039-0

26. Public Health England. Alcohol sales in England in 2014: Analysis to assess suitability for inclusion as an indicator in the Local Alcohol Profiles for England 2017. https://fingertips.phe.org.uk/documents/ Alcohol\%20sales\%20data\%202014.pdf

27. NatCen Social Research, Royal Free. and University College Medical School. Health Survey for England, 2011 [computer file]. Colchester,
Essex: UK Data Archive [distributor], April 2013. SN: 7260. Colchester, Essex: 2013. https://doi.org/10.5255/UKDA-SN-7260-1

28. Dutey-Magni $P$, Sinclair J, Brown J. Concurrent validity of an Estimator of Weekly Alcohol Consumption (EWAC) based on the Extended AUDIT 2018. https://doi.org/10.17605/OSF.IO/7WE4M

29. Boniface $S$, Kneale J, Shelton N. Drinking pattern is more strongly associated with under-reporting of alcohol consumption than sociodemographic factors: evidence from a mixed-methods study. BMC Public Health. 2014;14:1297. https://doi.org/10.1186/1471-245814-1297

30. Stockwell T, Donath S, Cooper-Stanbury M, Chikritzhs T, Catalano P, Mateo C. Under-reporting of alcohol consumption in household surveys: a comparison of quantity-frequency, graduated-frequency and recent recall. Addiction. 2004;99:1024-33. https://doi.org/10.1111/ j.1360-0443.2004.00815.x

31. Stockwell T, Zhao J, Macdonald S. Who under-reports their alcohol consumption in telephone surveys and by how much? An application of the 'yesterday method' in a national Canadian substance use survey. Addiction. 2014;109:1657-66. https://doi.org/10.1111/add. 12609

32. Stockwell T, Zhao J, Greenfield T, Li J, Livingston M, Meng Y. Estimating under- and over-reporting of drinking in national surveys of alcohol consumption: identification of consistent biases across four English-speaking countries. Addiction. 2016;111:1203-13. https:// doi.org/10.1111/add.13373

33. Lemmens $P$, Tan ES, Knibbe RA. Measuring quantity and frequency of drinking in a general population survey: a comparison of five indices. J Stud Alcohol. 1992;53(5):476-86. https://doi.org/10.15288/ jsa.1992.53.476

34. R Core Team. R: A language and environment for statistical computing. Vienna, Austria: R Foundation for Statistical Computing; 2017. https://www.R-project.org/

35. Wickham H, Averick M, Bryan J, Chang W, McGowan LD'A, François R, et al. Welcome to the Tidyverse. J. Open Source Softw. 2019;4(43):1686. http://doi.org/10.21105/joss.01686

36. Stan Development Team. RStan: The R interface to Stan 2018. http://mc-stan.org/

37. Dutey-Magni P. Concurrent validity of an estimator of weekly alcohol consumption (EWAC) based on the extended AUDIT. 2021. https://doi.org/10.5281/zenodo.5139511

38. Lavoie D. Alcohol identification and brief advice in England: a major plank in alcohol harm reduction policy. Drug Alcohol Rev. 2010;29(6):608-11. https://doi.org/10.1111/j.1465-3362.2010. 00224. $x$

39. DeLong ER, DeLong DM, Clarke-Pearson DL. Comparing the areas under two or more correlated receiver operating characteristic curves: a nonparametric approach. Biometrics. 1988;44:837-45. https://doi.org/10.2307/2531595

40. Brown J, West R, Angus C, Beard E, Brennan A, Drummond C, et al. Comparison of brief interventions in primary care on smoking and excessive alcohol consumption: a population survey in England. $\mathrm{Br} \mathrm{J}$ Gen Pract. 2016;66(642):e1-e9. https://doi.org/10.3399/ bjgp16X683149

41. Rehm J, Anderson P, Manthey J, Shield KD, Struzzo P, Wojnar M, et al. Alcohol use disorders in primary health care: what do we know and where do we go? Alcohol Alcohol. 2016;51(4):422-7. https:// doi.org/10.1093/alcalc/agv127

42. Mongan D, Long J. Standard drink measures throughout Europe. Peoples' understanding of standard drinks. RARHA: Joint Actional on Reducing Alcohol Related Harm; 2015. http://www.rarha.eu/ Resources/Deliverables/Lists/Deliverables/Attachments/14/WP5 \%20Background\%20paper\%20Standard\%20drink\%20measures\%20 HRB.pdf

43. Rosenberg G, Bauld L, Hooper L, Buykx P, Holmes J, Vohra J. New national alcohol guidelines in the UK: public awareness, 
understanding and behavioural intentions. J Public Health. 2018;40 (3):549-56. https://doi.org/10.1093/pubmed/fdx126

44. Rehm J, Anderson P, Gual A, Kraus L, Marmet S, Nutt D, et al. The tangible common denominator of substance use disorders: a reply to commentaries to Rehm et al. (2013a). Alcohol Alcohol. 2013;49(1): 118-22. https://doi.org/10.1093/alcalc/agt171

45. O'Donnell A, Abidi L, Brown J, Karlsson N, Nilsen P, Roback K, et al. Beliefs and attitudes about addressing alcohol consumption in health care: a population survey in England. BMC Public Health. 2018;18 (1):391. https://doi.org/10.1186/s12889-018-5275-2

46. UK Health. and Social Care Information Centre. UK SNOMED CT Drug Extension, RF2: Full, Snapshot \& Delta. 2018.

47. Haroon S, Wooldridge D, Hoogewerf J, Mittal A, Bhala N, O'Donnell A, et al. Information standards for recording alcohol use in electronic health records: Project report. London: Royal College of Physicians; 2018. https://www.rcplondon.ac.uk/file/ 10926/download

48. Rubinsky AD, Dawson DA, Williams EC, Kivlahan DR, Bradley KA. AUDIT-C scores as a scaled marker of mean daily drinking, alcohol use disorder severity, and probability of alcohol dependence in a U.S. general population sample of drinkers. Alcohol Clin Exp Res. 2013;37(8):1380-90. https://doi.org/10.1111/acer.12092

49. Frank D, DeBenedetti AF, Volk RJ, Williams EC, Kivlahan DR, Bradley KA. Effectiveness of the AUDIT-C as a screening test for alcohol misuse in three race/ethnic groups. J Gen Intern Med. 2008;23(6):781-87. https://doi.org/10.1007/s11606-0080594-0

50. Bradley KA, McDonell MB, Bush K, Kivlahan DR, Diehr P, Fihn SD. The AUDIT alcohol consumption questions: reliability, validity, and responsiveness to change in older male primary care patients. Alcohol Clin Exp Res. 1998;22(8):1842-49. https://doi.org/10.1111/j. 1530-0277.1998.tb03991.x

51. Heeb J-L, Gmel G. Measuring alcohol consumption: a comparison of graduated frequency, quantity frequency, and weekly recall diary methods in a general population survey. Addict Behav. 2005;30(3): 403-13. https://doi.org/10.1016/j.addbeh.2004.04.022
52. Livingston M, Callinan S. Underreporting in alcohol surveys: whose drinking is underestimated? J Stud Alcohol Drugs. 2015;76(1): 158-64. https://doi.org/10.15288/jsad.2015.76.158

53. Rehm J. Measuring quantity, frequency, and volume of drinking. Alcohol Clin Exp Res. 1998;22(s2):4s-14s. https://doi.org/10.1111/ j.1530-0277.1998.tb04368.x

54. Greenfield TK, Kerr WC, Bond J, Ye Y, Stockwell T. Improving graduated frequencies alcohol measures for monitoring consumption patterns: results from an Australian National Survey and a US diary validity study. Contemp Drug Probl. 2009;36(3-4):705-33. https:// doi.org/10.1177/009145090903600320

55. Hilton ME. A comparison of a prospective diary and two summary recall techniques for recording alcohol consumption. $\mathrm{Br} J$ Addict. 1989;84(9):1085-92. https://doi.org/10.1111/j.1360-0443.1989. tb00792.x

56. Jellinek EM. The disease concept of alcoholism. New Haven: Hillhouse Press; 1960. https://doi.org/10.1037/14090-000

57. Leggio L, Kenna GA, Fenton M, Bonenfant E, Swift RM. Typologies of alcohol dependence. From Jellinek to genetics and beyond. Neuropsychol Rev. 2009;19(1):115-29. https://doi.org/10.1007/ s11065-008-9080-z

\section{SUPPORTING INFORMATION}

Additional supporting information may be found in the online version of the article at the publisher's website.

How to cite this article: Dutey-Magni P, Brown J, Holmes J, Sinclair J. Concurrent validity of an estimator of weekly alcohol consumption (EWAC) based on the extended AUDIT. Addiction. 2021;1-10. https://doi.org/10.1111/add.15662 